\title{
Modificações no comportamento alimentar de crianças e adolescentes com leucemia: experiências de cuidadores familiares
}

\author{
Modifications in eating behavior of children and adolescents with leukemia: family \\ caregivers experience
}

Modificaciones de la conduta alimentaria de los niños y adolescentes con leucemia: la experiencia de los cuidadores familiares

Jéssica Carolina Del Frari ${ }^{1 *}$, Silvana Bastos Cogo ${ }^{1}$, Graciele Pontes ${ }^{1}$, Aline Ost dos Santos ${ }^{1}$, Fabiane Marzari Possatti ${ }^{1}$, Carolina Heleonora Pilger ${ }^{1}$, Graciela Dutra Sehnem¹.

\section{RESUMO}

Objetivo: Compreender a experiência dos cuidadores familiares face às modificações do comportamento e rotina alimentar de crianças e adolescentes em tratamento da leucemia. Métodos: Pesquisa qualitativa, do tipo descritiva e exploratória, realizada de julho a dezembro de 2020. Foi realizada entrevista semiestruturada analisada através da análise textual discursiva. Resultados: Emergiram as categorias: Caracterização sociodemográfica dos cuidadores familiares. Efeitos colaterais apresentados por crianças e adolescentes em tratamento para leucemia na perspectiva dos cuidadores familiares. Adaptações dos cuidadores familiares face as modificações no comportamento alimentar da criança e adolescente em tratamento para leucemia. Conclusão: Há necessidade de avaliar a aceitação do cardápio ofertado e traçar estratégias para melhorar a aceitação da alimentação hospitalar. É fundamental realizar ações educativas com os cuidadores familiares sobre a alimentação neste período de tratamento, além de disponibilizar um material impresso para que os mesmos tenham sucesso nesse desafio que são as mudanças no comportamento e na rotina alimentar de crianças e adolescentes em tratamento hemato-oncológico.

Palavras-chave: Comportamento alimentar, Leucemia, Criança, Cuidadores.

\begin{abstract}
Objective: To understand the experience of family caregivers facing modifications in the behavior and food routine of children and adolescents undergoing leukemia treatment. Methods: Qualitative, descriptive and exploratory research, carried out from July to December 2020. It was semi-structured interviews were applied and analyzed through textual discourse analysis. Results: The categories emerged: Family caregivers' sociodemographic characterization. Side effects presented by children and adolescents in leukemia treatment from the family caregivers' perspective. Adaptations of family caregivers face the changes in the feeding behavior of the child and adolescent in leukemia treatment. Conclusion: There is the need to evaluate the acceptance of the menu offered and to trace strategies to improve the acceptance of hospital feeding. It is essential to perform educational actions with family caregivers about feeding in this treatment period, in addition to providing printed material for them to succeed in this challenge, which are the changes in behavior and eating routine of children and adolescents in hemato-oncologic treatment.
\end{abstract}

Keywords: Feeding behavior, Leukemia, Child, Caregivers.

${ }^{1}$ Universidade Federal de Santa Maria, Santa Maria - RS. `E-mail: jeh.delfrari@hotmail.com

SUBMETIDO EM: 2/2022

ACEITO EM: 2/2022

PUBLICADO EM: $3 / 2022$ 


\section{RESUMEN}

Objetivo: Comprender la experiencia de los cuidadores familiares ante los cambios en el comportamiento y los hábitos alimentarios de los niños y adolescentes que reciben tratamiento contra la leucemia. Métodos: Investigación cualitativa, descriptiva y exploratoria, realizada de julio a diciembre de 2020. Se realizó entrevistas semiestructuradas que se analizaron mediante el análisis del discurso textual. Resultados: Surgieron las siguientes categorías: Caracterización sociodemográfica de los cuidadores familiares. Efectos secundarios que presentan los niños y adolescentes en tratamiento por leucemia desde la perspectiva de los cuidadores familiares. Adaptaciones de los cuidadores familiares frente a los cambios en el comportamiento alimentario del niño y el adolescente bajo tratamiento de la leucemia. Conclusión: Es necesario evaluar la aceptación del menú ofrecido y trazar estrategias para mejorar la aceptación de la alimentación hospitalaria. Es fundamental realizar acciones educativas con los cuidadores familiares sobre la alimentación en este periodo de tratamiento, además de proporcionarles material impreso para que tengan éxito en este reto, que son los cambios en el comportamiento y la rutina alimentaria de los niños y adolescentes en tratamiento hemato-oncológico.

Palabras clave: Conducta alimentaria, Leucemia, Niño, Cuidadores.

\section{INTRODUÇÃO}

O câncer infantojuvenil é considerado raro em comparação ao câncer no adulto e quando fora das possibilidades de cura os cuidados paliativos (CP) são a melhor opção (SILVA REG, et al., 2021). O processo de adoecimento e a rotina do tratamento oncológico são complexos, gerando sofrimento para o paciente e sua família (BICALHO C, et al., 2019). Em CP, a estratégia nutricional deve ser embasada nos princípios bioéticos bem como respeito as decisões do paciente e sua família (PAZ AS, et al., 2020).

O tratamento de doenças hematológicas como a leucemia tem itinerário terapêutico de longo prazo bem como recorrentes e prolongadas internações, consultas, procedimentos invasivos e dolorosos, tratamento agressivo e debilitante, gerando múltiplas mudanças emocionais, sociais, alimentares (CARLO MMRP e KUDO AM, 2018). Neste contexto, o diagnóstico de câncer em crianças e adolescentes impacta os cuidadores familiares e repercute na dinâmica familiar, podendo tornar-se uma experiencia desesperadora e dolorosa (PAULA DPS, et al., 2019).

Além deste sofrimento, há os efeitos colaterais comuns em pacientes pediátricos que recebem quimioterapia como: ansiedade, náuseas e vômitos (WONG CL, et al., 2021). Outro efeito é a neutropenia febril, tornando o paciente suscetível a infecções. Sob esta perspectiva, o rigor no preparo e higienização dos alimentos evita a contaminação do paciente (BAIOCCHI O, et al, 2018).

Diante das mudanças no comportamento alimentar de crianças e adolescentes em tratamento oncológico é relevante destacar o trabalho do nutricionista: suporte, orientações e estratégias nutricionais para amenizar os sintomas ocasionados pelo tratamento, de acordo com as especificidades, considerando os aspectos subjetivos do paciente (LOPES MS e CABRAL BEM, 2018).

É importante investigar como os cuidadores familiares experenciam às modificações do comportamento e da rotina alimentar de crianças e adolescentes em tratamento da leucemia. Este estudo tem por objetivo compreender a experiência dos cuidadores familiares face às modificações do comportamento alimentar de crianças e adolescentes em tratamento da leucemia.

\section{MÉTODOS}

Trata-se de pesquisa qualitativa, do tipo descritiva e exploratória, documental realizada em um hospital universitário referência nacional no Serviço de Oncologia Pediátrica. A unidade pesquisada atualmente possui dezoito leitos de internação, uma equipe de médicos, enfermeiros, nutricionista, fisioterapeuta, psicólogo e assistente social, além do serviço de secretariado e higienização. Assiste tanto pacientes portadores de doenças oncológicas, tais como neuroblastoma, nefroblastoma, tumor de wilms e ostessarcoma quanto 
doenças hematológicas, anemia falciforme, aplasias de medula, linfomas, púrpura trombocitopênica idiopática e outras. Dentre os tipos de câncer tratados na unidade pesquisada a leucemia é mais prevalente, por este motivo optou-se como objeto de pesquisa este público.

Este estudo tem como questão de pesquisa: como os cuidadores familiares experienciam as modificações do comportamento alimentar das crianças e adolescentes em tratamento da leucemia? Os participantes da pesquisa foram cuidadores familiares de crianças e adolescentes em tratamento de leucemia que estavam internadas na unidade de oncologia pediátrica. Estes foram selecionados por amostra de conveniência que objetiva reunir casos que possam contribuir para o aprofundamento da compreensão do fenômeno estudado (MOREIRA H e CALEFFE LG, 2008).

Foi realizado o convite para participar da pesquisa a doze cuidadores familiares, destes cinco participantes foram abordados, mas no momento encontravam-se impossibilitados de se ausentar do leito da criança ou adolescente, gerando a recusa na participação da pesquisa, obtendo-se um total de sete participantes. Cabe ainda destacar que devido a pandemia da COVID-19 foi necessário reduzir o número de internação na unidade limitando assim o público entrevistado.

Como critério de inclusão no estudo, cuidadores integrantes da família de crianças e adolescentes em tratamento da leucemia com idade igual ou superior a 18 anos. Considerou-se crianças até nove anos e adolescentes de 10 a 19 anos incompletos. Foram excluídos cuidadores familiares de crianças ou adolescentes em isolamento de contato bem como agravamento do quadro clínico da criança ou adolescente.

A coleta de dados aconteceu no período de julho a dezembro de 2020. A etapa inicial do estudo foi busca, por meio dos prontuários eletrônicos, de crianças e adolescente em tratamento da leucemia que atendessem aos critérios de inclusão no estudo. Foram selecionados crianças e adolescentes internadas no período da coleta. Após, o cuidador familiar foi convidado a participar, explicando os objetivos da pesquisa.

A obtenção dos dados aconteceu por meio de entrevista semiestruturada e questionamentos sobre as características sociodemográficas dos cuidadores familiares. As entrevistas foram gravadas por meio de gravador digital e posteriormente realizada a transcrição e dupla digitação dos dados, tendo uma média de duração de 42 minutos, com tempo mínimo de 23 minutos e máximo de 56 minutos. As entrevistas foram realizadas em sala reservada, assegurando a privacidade dos participantes e as medidas de biossegurança e distanciamento. Optou-se, devido a questões éticas, identificar os participantes da pesquisa com E para "entrevistado" somado a sequência alfanumérica do participante. As entrevistas foram analisadas a luz da Análise textual discursiva, sendo este um processo auto-organizado com a produção de novas compreensões em relação aos fenômenos estudados, sendo composta por quatro etapas, que são: desmontagem dos textos, estabelecimento de relações, captando o novo emergente e um processo auto-organizado (MORAES R e GALIAZZI MC, 2016).

A presente pesquisa fez parte do Projeto Guarda-Chuva intitulado "Experiência dos cuidadores familiares face às modificações comportamentais da criança e adolescente com leucemia" obedeceu a Resolução 466/12 e 510/16, regulamentada pelo Conselho Nacional de Saúde (CNS), que normatiza e autoriza a pesquisa em seres humanos, obtendo autorização da instituição para aplicação e sendo submetido ao Comitê de Ética em Pesquisa da Universidade Federal de Santa Maria Envolvendo Seres Humanos (CEP) da UFSM, sendo aprovado com registro CAAE número 32139020.7.0000.534.

\section{RESULTADOS}

\section{Caracterização sociodemográfica dos cuidadores familiares.}

Participaram do estudo sete cuidadores familiares com idade entre 26 a 45 anos (média de 33,4 anos), destes seis eram mulheres e mães das crianças e/ou adolescentes em tratamento da leucemia. Quando analisado o estado civil dos participantes, cinco se declararam casados (71,4\%). As profissões dos participantes do estudo são: enfermeira (14,3\%), instrutor de MMA $(14,3 \%)$, agricultor $(14,3 \%)$, autônomo $(14,3 \%)$, secretária $(14,3 \%)$, gerente administrativo $(14,3 \%)$. Os níveis de escolaridade são: ensino 
fundamental incompleto $(28,6 \%)$, ensino médio incompleto $(14,3)$, ensino médio completo $(42,8 \%)$, ensino superior complete $(14,3)$. Dentre os principais cuidadores das crianças e adolescentes estão a mãe $(85,7 \%)$, o pai $(14,3 \%)$, sendo relatado também o auxílio no cuidado por irmã e avós maternos.

As crianças e adolescentes em tratamento da leucemia possuíam idade entre dois e 18 anos (média de 7,14 anos), destes quatro do sexo feminino e três do sexo masculino, destes (71, 4\%) com Leucemia Linfocítica Aguda (LLA) e 28,6\% com Leucemia Mielóide Aguda (LMA), com predominância do diagnóstico de LLA em fase de consolidação $(85,7 \%)$ e recidiva $(14,3)$.

\section{Efeitos colaterais apresentados por crianças e adolescentes em tratamento para leucemia na perspectiva dos cuidadores familiares.}

Alguns dos efeitos colaterais observados pelos cuidadores familiares entrevistados:

"Muitos (efeito da medicação na alimentação), principalmente das quimios, é falta de apetite, medo de passar mal dos enjoos, no caso os enjoos, ou então por causa dos corticoides que dói a barriga, que começa a doer a barriga depois que ela se alimenta (...)" (E2).

"Feridas na boquinha, as náuseas, os vômitos (...) atrapalhou (o apetite). Quando ela estava fazendo as quimios, ela comia e vomitava. Não conseguia com o cheiro da comida, depois que parou, ela tinha vontade de comer, só que não conseguia por causa das feridas na boca" (E7).

Outro efeito colateral relatado pelos entrevistados foi a alteração de paladar que os levou a não consumir alimentos que antes eram rotina na sua alimentação e a introduzir alimentos que não eram ingeridos.

"Mamão ele sentava e comia, agora ele não come mais. Beterraba, eu cozinhava, ele sentava e comia, não come mais, cenoura, que ele nunca gostou de comer... agora quando vem ele come a travessinha cheia, cenoura pura (...) coisas que ele nunca...não gostava antes, agora ele quer comer bastante, cada dia uma surpresa" (E5).

Os cuidadores familiares demonstram preocupação e sensação de impotência com a influência dos efeitos colaterais na alimentação das crianças e adolescentes.

“(...) Queria eu estar passando, não ela (...) Eu me sentia horrível, meu Deus, não sei nem explicar. Tu ver tua filha ali, ela não conseguia nem tomar água direito. Ver tua filha ali, querendo comer e não conseguir, querendo tomar água e não conseguir, passando 24 horas com aquela dor. Ela tomava remédio para dor, tomou até morfina, mas a dor mesmo assim não passava" (E7).

Diante dos relatos dos cuidadores familiares é possível compreender que o tratamento da leucemia ocasiona diversos efeitos colaterais. Estes influenciam diretamente na alimentação dos pacientes, desencadeando mudanças no comportamento alimentar das crianças e adolescentes em tratamento oncológico.

\section{Adaptações dos cuidadores familiares face as modificações no comportamento alimentar da criança e adolescente em tratamento para leucemia.}

De acordo com os relatos dos cuidadores familiares as crianças e adolescentes apresentaram mudanças em seu comportamento alimentar após o início do tratamento, como por exemplo os relacionados com o aumento na diversidade dos alimentos consumidos e suas preferências alimentares:

"Ele foi uma criança que nunca foi de se alimentar muito bem, até os dois, três anos de idade era basicamente o leite, (...) não era muito diversificada a dieta dele, mas chegando aqui, na primeira fase, teve a questão dos corticoides, acabou que ele começou a provar outras coisas e aí aumentou um pouquinho a... faltou a palavra agora, mas aumento o número de coisas que ele comia" (E1). 
Outra mudança observada pelos cuidadores familiares foi o aumento do apetite pelas crianças e adolescentes.

"A primeira vez que ela internou, ela não comia praticamente nada. Agora ela está comendo mais. Comendo bem mais. Aumentou o apetite parece" (E7).

Embora os relatos mostrem o aumento na diversidade alimentar e aumento do apetite, os cuidadores familiares notaram que durante a permanência dos pacientes no hospital eles não apresentaram uma boa aceitação da alimentação ofertada e seu domicílio eles aceitam melhor as refeições quando comparado ao hospital.

"Só aqui, por que em casa ela come, de manhã ela acorda e come duas torradas, ela almoça bem, ela come brócolis, ela come tomate, beterraba, alface, tipo, ela adora comida (...)" (E4).

A hospitalização e o tratamento acabam influenciando a alimentação em casa, pois em alguns casos as crianças acabam recusando também no domicílio os alimentos que são ofertados durante a internação e os cuidadores acabam tendo que encontrar estratégias para manter a oferta alimentar no domicílio alterando a forma de preparo ou oferecendo alimentos diferentes dos consumidos no hospital.

"Ela parou de comer bastante coisa que ela comia. Feijão que ela adorava, ela não quer, ela enjoou. A lentilha também ela não quer, ela enjoou (...) eu acho, gurias, que de repente é porque vem aqui sabe. Quando eu faço assim, vou com o prato, cheiro bom não, mãe, ela diz. Que cheiro bom é a comida. Ela não come mesmo. Então eu tento adequar, adaptar outras coisas para ela comer que não lembra ela daqui" (E6).

Os cuidadores familiares relataram que após o início do tratamento o nutricionista orientou sobre os cuidados em domicílio e entregou um material informativo.

"As orientações de alimentação, higiene e alimentação, essas coisas, foi o nutricionista" (E1).

“(...) a nutricionista, até ela deu uma cartelinha para levar com o que ele podia comer e o que não podia comer (...)" (E2).

Os cuidadores familiares inferiram que foram necessárias alterações na rotina dessas famílias após o início do tratamento das crianças e adolescentes com leucemia:

"(...) antes eu cozinhava qualquer coisa, qualquer coisa para ela estava bom, e agora eu levanto de manhã cedo e a primeira coisa que eu faço é perguntar para ela o que ela quer comer, (...) às vezes eu faço alguma coisa diferente para o irmão dela e para o pai, mas para ela eu tenho que primeiramente buscar ela para ver o que ela quer se alimentar no dia, o que ela tem vontade, porque aqui dentro ela tem que comer o que vem. Então quando ela está lá fora eu busco fazer o que ela quer comer, porque é pouco tempo que ela fica lá fora (...)" (E3).

Há que se destacar manifestações relacionadas aos cuidados com a higiene dos alimentos, bem como mudanças de hábitos.

“(...) antes eu não tinha tanto cuidado com a higiene dos alimentos, até a rotina do trabalho da gente era meio corrido. Tu pegava no mercado, passava uma água ali, temperava e deu. Agora não, tem que ter todo aquele cuidado, de higienizar antes, tal e tal coisa. Antes de guardar e tudo. E gente, também comecei a fazer mais saladas, comer mais coisas saudáveis (...)" (E6).

Os cuidadores familiares relatam que as alterações desencadeadas na rotina da família influenciam em muitos aspectos da sua vida, que vão além da alimentação, pois eles necessitam procurar estratégias para diminuir os efeitos colaterais do tratamento e reorganizar o cotidiano. 


\section{DISCUSSÃO}

Os cuidadores familiares entrevistados eram em sua maioria do sexo feminino, mães das crianças ou adolescentes em tratamento da leucemia. Um estudo transversal com 163 pais responsáveis de crianças oncológicas, no Serviço de Oncologia Pediátrica, a mãe é a responsável pelos cuidados (81\%), com idade entre 25 a 34 anos (44,8\%) (PEDROSA BRV, et al., 2019). Um estudo descreveu as experiências de pais e mães de crianças e adolescentes com leucemia na transição dos cuidados do hospital para o domicílio e concluiu que eles precisaram se adaptar à nova realidade de cuidados bem como realizar o registro das informações corrobora para a compreensão dos pais sobre a doença, o tratamento, as possíveis estratégias para evitar efeitos adversos e novas internações (RODRIGUES FMS, et al., 2019).

A leucemia é uma condição desafiadora para paciente e família, afetando as dimensões física, social e psicológica do paciente e seu cuidador. O câncer infanto-juvenil é complexo, necessitando da percepção diferenciada da equipe interdisciplinar, considerando a clínica ampliada (SOUZA RLA, et al., 2021). A intervenção nutricional contínua auxilia a redução dos sintomas e declínio funcional do paciente oncológico (FERREIRA PF, et al., 2021).

O nutricionista tem papel de prestar suporte ao paciente durante o tratamento, através uma terapia nutricional adequada e do fornecimento de informações sobre estratégias nutricionais que podem ser utilizadas para amenizar os sintomas ocasionados pelo tratamento, considerando a individualidade de cada criança ou adolescente e abordando as suas necessidades nutricionais, restrições alimentares, tolerância da alimentação, estado clínico e os efeitos colaterais apresentados (LOPES MS e CABRAL BEM, 2018).

As dificuldades na alimentação como a recusa de se alimentar, ingestão seletiva com o consumo de pouca quantidade e/ou da variedade de alimentos e a aversão à alimentação tem impacto na nutrição do paciente, interferindo no seu estado nutricional. O tratamento medicamentoso oncológico tem o potencial de interferir no paladar como hipogeusia, disgeusia, ageusia e hipergeusia (SILVA EHS, et al., 2021).

Em um estudo que analisou a evolução do estado nutricional de 50 adolescentes de 10 a 19 anos após tratamento oncológico para leucemias e linfomas, evidenciando a necessidade de novos estudos além de, em campo prático, avaliar e realizar intervenções nutricionais ao longo do tratamento para a promoção de do estado nutricional adequado em adolescentes sobreviventes à leucemia (BELIN CHS, et al., 2019).

O tratamento da leucemia ocasionou efeitos colaterais indesejáveis nas crianças e adolescentes deste estudo, impactando o comportamento alimentar dos pacientes e indiretamente, na rotina da família. Os principais efeitos colaterais apresentados pelas crianças e adolescentes relatados pelos cuidadores familiares foram náusea, vômito, mucosite oral, perda de apetite, também conhecido por inapetência alimentar e alteração de paladar. Sob essa perspectiva, os efeitos colaterais dos quimioterápicos são atribuídos ao potencial de destruição das células que acabam por atingir também as células sadias do organismo, principalmente células do trato gastrointestinal, causando impacto diretamente na alimentação (SOUSA GS, et al., 2018).

Os cuidadores familiares entrevistados demonstram preocupação e sensação de impotência diante dos efeitos colaterais do tratamento que os pacientes apresentaram, pois estes interferem na alimentação, causando dor e desconforto ao se alimentar. Há a afirmação que preferiam estar no lugar da criança ou adolescente para que eles não precisassem sofrer diante do processo de adoecimento. Os achados são comprovados pela literatura, uma vez que os CP aliviam os sintomas que impactam a qualidade de vida (DIAS KCC, et al., 2020).

Os cuidadores familiares destacaram mudanças no comportamento alimentar de crianças e adolescentes após o início do tratamento da leucemia, através do aumento do apetite e da diversidade dos alimentos consumidos, assim como mudança em suas preferências alimentares, a partir da disponibilidade dos alimentos no cardápio ofertado pelo hospital para que eles possam (re)experimentar. Sob esta perspectiva, uma pesquisa com nove profissionais da equipe multiprofissional de um serviço de oncologia pediátrica evidenciou que o cuidado seguro pode ocorrer mediante orientações de diferentes profissionais, sendo fundamental o planejamento e orientações, disponibilizando a educação dessas famílias (KUNTZ SR, et al., 2021). 
Para tanto, a disponibilização de um material informativo, com orientações a respeito dos cuidados necessários nessa etapa se torna um importante recurso de educação em saúde, facilitando a mediação de conteúdo para o cuidador, que poderá utilizá-lo quando necessário para esclarecimento de dúvidas, diminuindo as preocupações dos familiares, as intercorrências e reinternações das crianças ou adolescentes (COSTA CIA, 2019).

Sob esta perspectiva, o desenvolvimento do material educativo deve ser simples, apresentando informações claras e precisas (SILVA REG, et al, 2021). Cartilhas educativas são recursos que podem ser desenvolvidos com orientações para os familiares pela equipe interdisciplinar. As tendências de produções oriundas de dissertações e teses brasileiras, sobre cuidados paliativos em oncologia pediátrica sugeriu novos estudos para aprofundamento teórico (DIAS KCC, et al., 2020).

\section{CONCLUSÃO}

O processo terapêutico da doença acarreta mudanças no comportamento alimentar da criança ou adolescente em tratamento da leucemia que repercute na rotina alimentar da sua família. Entender como os cuidadores experienciam essa etapa possibilita visualizar meios que os auxiliem a lidar com as mudanças no comportamento alimentar deste público e identificar possíveis comportamentos de risco, que interfiram no estado nutricional da criança ou adolescente e no tratamento e os ajudem a traçar estratégias para lidar com essas alterações. Para tanto, há necessidade de avaliar a aceitação do cardápio ofertado e traçar estratégias para melhorar a aceitação da alimentação hospitalar. Sob esta perspectiva, é fundamental realizar ações educativas com os cuidadores familiares sobre a alimentação neste período de tratamento, além de disponibilizar um material impresso para que os mesmos tenham sucesso nesse desafio que são as mudanças no comportamento e na rotina alimentar de crianças e adolescentes em tratamento hematooncológico.

\section{REFERÊNCIAS}

1. BAIOCCHI O, et al. Aspectos nutricionais em oncologia. 1, ed. Rio de Janeiro: Atheneu, 2018. $628 \mathrm{p}$.

2. BELIN CHS, et al, 2019. Changes in nutritional status in adolescents surviving leukemia and lymphoma. Revista de Nutrição; 2020;33: e190194.

3. BICALHO C, et al. Processo de adolescer relacionado ao adoecimento e tratamento do câncer. Psic Saúde Doenças, 2019; 1:74-87.

4. CARLO MMRP, KUDO AM. Terapia Ocupacional em Contextos Hospitalar e Cuidados Paliativos. São Paulo: Editora Payá, 2018. 448p.

5. COSTA CIA. Construção de uma cartilha educativa para familiares de crianças com leucemia para o cuidado domiciliar. Dissertacão (Mestrado em Enfermaqem) - Universidade do Estado do Rio de Janeiro, 2019. 1124p.

6. DIAS KCC, et al. Dissertações e teses sobre cuidados paliativos em oncologia pediátrica: estudo bibliométrico. Acta Paulista de Enfermaqem; 2020;33: eAPE20190264.

7. FERREIRA PF, et al. Declínio do estado nutricional infanto-juvenil durante o tratamento oncológico: seus agravantes e relevância nutricional. Research Society and Development; 2021; 10:e481101624229.

8. KUNTZ SR, et al. Primeira transicão do cuidado hospitalar para domiciliar da criança com câncer: orientações da equipe multiprofissional. Escola Anna Nery; 2021;25: e20200239.

9. LOPES MS CABRAL, BEM. Terapia nutricional na promoção do ganho de peso de pacientes em tratamento quimioterápico. Revista Científica da Faminas, 2018; 13: 5-11.

10. MORAES R, GALIAZZI MC. Análise Textual Discursiva. 2. ed. liuí: Editora Uniiuí, 2016. 264p.

11. MOREIRA H, CALEFFE LG. Metodologia da pesquisa para o professor pesquisador. Rio de Janeiro: DP\&A, 2008.248 p.

12. PAULA DPS, et al. Câncer infantojuvenil do âmbito familiar: percepções e experiências frente ao diagnóstico. Rev Cuidarte, 2019, 10: e570.

13. PAZ AS, et al. Nutrição em cuidados paliativos oncológicos: Aspectos bioéticos. Brazilian Journal of Health Review; 2020;4: $8891-903$.

14. PEDROSA BRV, et al. Parents' knowledge about the oral health care of oncological children. RGO - Revista Gaúcha de Odontologia; 2019;67.

15. RODRIGUES FMS, et al. Transição de cuidados para o domicílio na perspectiva de pais de filhos com leucemia. Revista Gaúcha de Enfermaqem. 2019, 40: e20180238.

16. SILVA REG, et al. Validation of an exercise booklet for children with acute lymphoblastic leukemia. Fisioterapia em Movimento; 2021;34.

17. SILVA EHS, et al., 2021. Alterações no paladar advindos de quimioterapia convencional. Research Society and Development; 2021; 10: e589101422467.

18. SOUSA GS, et al. Repercussão do ciclo quimioterápico no estado nutricional de crianças e adolescentes em Teresina-PI. Revista Interdisciplinar, 2018, 11:67-74.

19. SOUZA RLA, et al. Hospitalization perceived by children and adolescents undergoing cancer treatment. Revista Gaúcha de Enfermaqem; 2021:42: e20200122.

20. WONG CL, et al. Effects of immersive virtual reality for preventing and managing anxiety, nausea and vomiting amona pediatric cancer patients receiving their first chemotherapy: A study protocol for an exploratory trial. Plos one, 2021;10: e0258514. 